\title{
Documento de posicionamiento de la Asociación Española de Pediatría-Sociedad Española de Infectología Pediátrica sobre el tratamiento de las infecciones por bacterias multirresistentes
}

\author{
Position statement of the Spanish Association of Pediatrics-Spanish Society of \\ Pediatric Infectology on the treatment of multidrug-resistant bacterial infections
}

David Aguilera-Alonso, ${ }^{*}$ Luis Escosa-García, ${ }^{\ddagger}$ Walter Alfredo Goycochea-Valdivia, ${ }^{\S}$

Pere Soler-Palacín," Jesús Saavedra-Lozano, *,"l Carlos Rodrigo, ${ }^{* *}$

Emilia Cercenado, ${ }^{\ddagger},{ }^{\S}$ José Tomás Ramos, ${ }^{\S}$ Fernando Baquero-Artigao

* Sección de Enfermedades Infecciosas, Servicio de Pediatría, Instituto de Investigación Sanitaria, Hospital General Universitario Gregorio Marañón, Madrid, España.

₹ Servicio de Pediatría, Enfermedades Infecciosas y Patología Tropical, Hospital La Paz, Universidad Autónoma de Madrid, Madrid, España.

$\S$ Servicio de Inmunología, Reumatología e Infectología Pediátrica, Hospital Universitario Virgen del Rocío, Sevilla, España.

"l Unitat de Patologia Infecciosa i Immunodeficiències de Pediatria, Hospital Vall d’Hebron, Universidad Autónoma de Barcelona, Barcelona, España. ๆ Universidad Complutense de Madrid, España.

** Servicio de Pediatría, Hospital Germans Trias i Pujol, Universidad Autónoma de Barcelona, Barcelona, España.

¥¥ Servicio de Microbiología y Enfermedades Infecciosas, Hospital General Universitario Gregorio Marañón, Universidad Complutense de Madrid, Madrid, España. CIBERES, Centro de Investigación Biomédica en Red de Enfermedades Respiratorias, CB06/06/0058, Madrid, España.

$\S \S$ Servicio de Pediatría, Hospital Clínico San Carlos, Universidad Complutense de Madrid, Madrid, España.

RESUMEN

En los últimos años se ha evidenciado un incremento en la incidencia de infecciones por bacterias multirresistentes. Las principales amenazas son los bacilos gramnegativos productores de $\beta$-lactamasas de espectro extendido (BLEE), AmpC o carbapenemasas, Staphylococcus aureus resistente a la meticilina y Enterococcus faecium resistente a la vancomicina. Para hacer frente a este problema es fundamental establecer programas de optimización en el uso de antimicrobianos (PROA) específicos para pediatría, realizar una vigilancia epidemiológica activa y desarrollar una adecuada política de control de infecciones. Su abordaje terapéutico es, a menudo, complejo y multidisciplinar, precisando frecuentemente el uso de antibióticos con menor experiencia. En este documento de posicionamiento, elaborado por la Asociación Española de Pediatría y la Sociedad Española de Infectología Pediátrica, se revisa la epidemiología y el tratamiento de estas infecciones siguiendo la mejor evidencia disponible.

Palabras clave: Carbapenemesas, multidrogorresistencia, infecciones, tratamiento, ßeta lactamasa de espectro extendido.

\section{ABSTRACT}

Recent years have seen an increase in the incidence of infections by multi-drug resistant bacteria. The main threats are the Gram-negative bacilli that produce $\beta$-spread-spectrum lactamases (BLEE), AmpC or carbapenemases, methicillinresistant Staphylococcus aureus and vancomycin-resistant Enterococcus faecium. To address this problem, it is essential to establish antimicrobial use optimization programs $(A O P)$ specific to pediatrics, conduct active epidemiological surveillance and develop an appropriate infection control policy. Its therapeutic approach is often complex and multidisciplinary, often requiring the use of less experienced antibiotics. This position paper, prepared by the Spanish Association of Pediatrics and the Spanish Society of Pediatric Infectious Diseases, reviews the epidemiology and treatment of these infections according to the best available evidence.

Keywords: Carbapenemases, multi-drug resistant, infections, treatment, extended-spectrum $\beta$ eta lactamases.

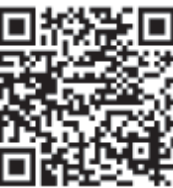




\section{INTRODUCCIÓN}

La resistencia a antimicrobianos supone, actualmente, uno de los principales problemas de salud pública. ${ }^{1}$ La prevalencia de bacterias multirresistentes (BMR) se ha incrementado en las últimas décadas de forma significativa.

El tratamiento de las infecciones por BMR puede implicar también la utilización de antibióticos con menor experiencia. La mayoría de la evidencia actual procede de estudios observacionales realizados en adultos, ${ }^{2,3}$ y muy frecuentemente nos encontramos con la necesidad de recurrir al uso off-label de los escasos antimicrobianos disponibles.

El tratamiento debe individualizarse según la gravedad, las características del paciente, el foco de la infección y el patrón de sensibilidad de las bacterias aisladas. Además del tratamiento antimicrobiano, son prioritarios el tratamiento de soporte y el control del foco, siempre que sea posible. En las Tablas 1 y 2 se muestran las dosis de los principales antibióticos utilizados en las infecciones por BMR. La duración del tratamiento antibiótico ha de ajustarse a la máxima evidencia disponible. ${ }^{4}$

\section{INFECCIONES POR COCOS GRAMPOSITIVOS}

\section{Staphylococcus aureus resistente a meticilina}

El principal mecanismo de resistencia es la adquisición de una nueva proteína de unión a la penicilina, denominada PBP2a, que está codificada por el gen $m e c A,{ }^{5}$ la cual ocasiona resistencia a la mayoría de $\beta$-lactámicos, con la excepción de las nuevas cefalosporinas (ceftarolina y ceftobiprol).

\section{Epidemiología}

Actualmente, estas cepas suponen entre $5-10 \%$ de las infecciones por $S$. aureus en niños en España, llegando a ser de $25 \%$ en el caso de neumonías adquiridas en la comunidad (NAC). ${ }^{6}$ La mayoría de los casos comunicados son infecciones de piel y partes blandas (IPPB), seguidas de neumonías y bacteriemias.

Aunque en otros países europeos su prevalencia ha disminuido progresivamente en los últimos años, en España se mantiene estable, situándose en torno a $12 \%$ los aislamientos en hemocultivo en niños, según los datos del Centro Europeo de Control de Enfermedades (ECDC).

\section{Tratamiento}

El adecuado control del foco debe considerarse el primer escalón y una prioridad absoluta para un tratamiento eficaz (Tabla 3). En caso de bacteriemia persistente a pesar de un tratamiento correcto, debe realizarse una búsqueda activa de focos a distancia.

Los glucopéptidos (vancomicina o teicoplanina) se han posicionado como los antibióticos de referencia para el tratamiento de infecciones por $S$. aureus resistente a meticilina (SARM). Sin embargo, su alta unión a proteínas plasmáticas limita su difusión a tejidos. Por ello, es fundamental la monitorización de las concentraciones plasmáticas con el fin de garantizar valores adecuados para el tratamiento de infecciones graves.

Entre las opciones disponibles, la clindamicina y el linezolid inhiben la síntesis de toxinas, por lo que su uso en monoterapia o combinado con otros antibióticos puede ser útil en el caso de infecciones por cepas productoras de toxinas. El linezolid presenta muy buena difusión a tejidos y permite realizar terapia secuencial de vía intravenosa a oral, aunque puede presentar toxicidad hematológica y neurológica en tratamientos prolongados.

La rifampicina presenta una excelente actividad frente al biofilm, siendo recomendable asociarlo al tratamiento antibiótico de elección en el caso de infecciones por $S$. aureus relacionadas con material protésico. ${ }^{7}$ El cotrimoxazol mantiene en la actualidad actividad frente a la mayoría de aislamientos de SARM, siendo una buena opción en infecciones leves o moderadas.

En bacteriemia o endocarditis por SARM con una concentración mínima inhibitoria (CMI) de vancomicina $\geq 1.5 \mathrm{mg} / \mathrm{L}$, una buena opción es la daptomicina. Este antibiótico es bactericida, está aprobado en niños mayores de un año y se administra en una sola dosis diaria. Como aspectos negativos, destaca su inactivación por el surfactante pulmonar.

La ceftarolina es una cefalosporina de quinta generación aprobada desde el nacimiento para el tratamiento de IPPB y NAC, con excelente perfil de seguridad y buena actividad frente a SARM ${ }^{8}$ aunque la experiencia en niños es aún limitada.

\section{Enterococcus spp.}

Los microorganismos del género Enterococcus se caracterizan por presentar resistencia intrínseca a múltiples antimicrobianos, incluyendo casi todas 
Tabla 1: Dosis de los principales antibióticos utilizados en pediatría para el tratamiento de infecciones por cocos grampositivos resistentes.

\begin{tabular}{|c|c|c|c|}
\hline Antibiótico & Dosis estándar & Dosis específica (1) & Comentarios \\
\hline Ampicilina (IV) & $\begin{array}{l}\geq 1 \mathrm{~m}: 100-200 \mathrm{mg} / \mathrm{kg} / \mathrm{día} \text { c/6 horas } \\
\text { (dosis máx.: } 12 \mathrm{~g} / \mathrm{día} \text { ) } \\
<1 \mathrm{~m} \text { : consultar dosis según peso, } \\
\text { EG y EPN }\end{array}$ & $\begin{array}{l}\text { En el caso de infecciones graves } \\
\text { (p. ej., meningitis o endocarditis): } \\
300-400 \mathrm{mg} / \mathrm{kg} / \mathrm{dí} \text { c/4-6 horas } \\
\text { (dosis máx.: } 12 \mathrm{~g} / \text { día) }\end{array}$ & $\begin{array}{l}\text { Valorar el uso de la dosis más alta } \\
\text { c/4h (máx.: } 24 \text { g/día) en infecciones } \\
\text { por Enterococcus spp. con CMI } \\
16-64 \text { mg/L sin producción de } \\
\beta \text {-lactamasas }\end{array}$ \\
\hline Vancomicina (IV) & $\begin{array}{l}\geq 1 \mathrm{~m}: 45 \mathrm{mg} / \mathrm{kg} / \mathrm{dí} a \mathrm{c} / 6-8 \text { horas } \\
\text { (dosis máx. inicial: } 4 \mathrm{~g} / \mathrm{día} \text { ) } \\
<1 \mathrm{~m} \text { : consultar dosis según peso, } \\
\text { EG y EPN }\end{array}$ & $\begin{array}{l}\text { Utilizar } 15 \mathrm{mg} / \mathrm{kg} / \text { dosis} / 6 \mathrm{hrs} \text { (dosis } \\
\text { máx. inicial: } 4 \mathrm{~g} / \mathrm{día} \text { ) en bacterie- } \\
\text { mia, meningitis, IOA, o } \mathrm{S} \text {. aureus } \\
\text { con CMl de vancomicina > } 1 \mathrm{mg} / \mathrm{L} \text {. } \\
\text { Se puede administrar en perfusión } \\
\text { continua en el caso de infecciones } \\
\text { graves }\end{array}$ & $\begin{array}{l}\text { Concentración plasmática objetivo: } \\
\text { valle }>10 \mu \mathrm{g} / \mathrm{mL} \text {. En el caso de } \\
\text { infecciones graves o } \mathrm{S} \text {. aureus } \\
\text { con } \mathrm{CMI} \text { de vancomicina }>1 \mathrm{mg} / \mathrm{L} \text { : } \\
15-20 \mu \mathrm{g} / \mathrm{mL} \\
\text { Concentraciones plasmáticas } \\
\text { objetivo en perfusión continua: } \\
20-25 \mu \mathrm{g} / \mathrm{mL}\end{array}$ \\
\hline Teicoplanina (IV) & $\begin{array}{l}\geq 2 \mathrm{~m}: 10 \mathrm{mg} / \mathrm{kg} / \mathrm{dosis} \mathrm{c} / 12 \text { horas } \\
\text { (3 dosis) y luego c/24 horas } \\
<2 \mathrm{~m} \text { : dosis de carga de } 16 \mathrm{mg} / \\
\mathrm{kg} \text { el primer día seguido de } 8 \mathrm{mg} / \\
\mathrm{kg} / 24 \text { horas } \\
\text { Máx.: } 400 \mathrm{mg} / \text { dosis }\end{array}$ & $\begin{array}{l}\text { Considerar } 12 \text { mg/kg/dosis en } \\
\text { infecciones graves, bacteriemia o } \\
\text { endocarditis (máx.: } 800 \text { mg/dosis) }\end{array}$ & $\begin{array}{l}\text { Escasa penetración en LCR } \\
\text { Considerar alternativas en casos } \\
\text { con concentraciones plasmáticas } \\
\text { impredecibles (p.ej., hipoalbumi- } \\
\text { nemia grave o insuficiencia renal } \\
\text { crónica) }\end{array}$ \\
\hline Ceftarolina (IV) & $\begin{array}{l}\text { Infusión IV en } 1 \text { hora: > } 33 \text { kg: } 600 \\
\text { mg/12 horas } \\
>2 \text { a: } 12 \text { mg/kg/8 horas (máx.: } 400 \\
\text { mg/8 horas) } \\
2 \text { m-2 a: } 8 \text { mg/kg/8 horas } \\
\text { < } 2 \text { m: } 6 \mathrm{mg} / \mathrm{kg} / 8 \text { horas }\end{array}$ & $\begin{array}{l}\text { En niños } \geq 6 \text { años con FQ y exa- } \\
\text { cerbación respiratoria considerar } \\
15 \text { mg/kg/8h (máx.: } 600 \text { mg/dosis) }\end{array}$ & Aprobado para IPPB y NAC \\
\hline Linezolid (IV o VO) & $\begin{array}{l}>12 \text { a: } 600 \mathrm{mg} / 12 \text { horas } \\
\leq 12 \text { a: } 10 \mathrm{mg} / \mathrm{kg} / 8 \text { horas (máx.: } \\
600 \mathrm{mg} / \text { dosis) }\end{array}$ & & $\begin{array}{l}\text { Aprobado para IPPB y NAC. Uso } \\
\text { off-label en edad pediátrica en } \\
\text { Europa }\end{array}$ \\
\hline Daptomicina (IV) & $\begin{array}{l}>11 \text { a: } 5 \mathrm{mg} / \mathrm{kg} / 24 \text { horas } \\
7-11 \text { a: } 7 \mathrm{mg} / \mathrm{kg} / 24 \text { horas } \\
1-6 \text { a: } 10 \mathrm{mg} / \mathrm{kg} / 24 \text { horas }\end{array}$ & $\begin{array}{l}\text { Considerar dosis mayores en } \\
\text { infecciones graves, bacteriemia y } \\
\text { endocarditis: } \\
\text { > } 11 \text { a: } 8-10 \mathrm{mg} / \mathrm{kg} / 24 \text { horas } \\
\text { 7-11 a: } 9 \mathrm{mg} / \mathrm{kg} / 24 \text { horas } \\
1-6 \text { a: } 12 \mathrm{mg} / \mathrm{kg} / 24 \text { horas }\end{array}$ & $\begin{array}{l}\text { No utilizar en infecciones pulmona- } \\
\text { res debido a su inactivación por el } \\
\text { surfactante pulmonar } \\
\text { La dosis máx. no ha sido } \\
\text { establecida } \\
\text { Aprobado en } \geq 12 \text { m para IPPB } \\
\text { y endocarditis/bacteriemia por } S \text {. } \\
\text { aureus. Se desaconseja su uso en } \\
<12 \mathrm{~m} \text {, salvo falta de otras alterna- } \\
\text { tivas disponibles }\end{array}$ \\
\hline $\begin{array}{l}\text { Cotrimoxazol (trimeto- } \\
\text { prima-sulfametoxazol } \\
\text { (IV o VO) }\end{array}$ & $\begin{array}{l}\text { Trimetoprima 8-15 mg/kg/día c/8-12 } \\
\text { horas (VO) o c/6-12 horas (IV) } \\
\text { Máx.: trimetoprima } 320 \mathrm{mg} / 6 \text { horas }\end{array}$ & $\begin{array}{l}\text { En el caso de infecciones ostear- } \\
\text { ticulares considerar la dosis más } \\
\text { elevadas }\end{array}$ & $\begin{array}{l}\text { Evitar en el tratamiento inicial de } \\
\text { infecciones graves }\end{array}$ \\
\hline $\begin{array}{l}\text { Clindamicina (IV o } \\
\text { VO) }\end{array}$ & $\begin{array}{l}\geq 1 \mathrm{m:} 30-40 \mathrm{mg} / \mathrm{kg} / \mathrm{día} \text { c/6-8 horas } \\
<1 \mathrm{m:} 15-20 \mathrm{mg} / \mathrm{kg} / \mathrm{día} \mathrm{c} / 6-8 \text { horas } \\
\text { Máx.: VO } 1.8 \mathrm{~g} / \mathrm{día} \text {; IV } 2.7 \mathrm{~g} / \mathrm{día}\end{array}$ & $\begin{array}{l}\text { En el caso de infecciones } \\
\text { moderadas-graves utilizar las dosis } \\
\text { más altas }\end{array}$ & $\begin{array}{l}\text { En el caso de resistencia in vitro } \\
\text { a la eritromicina, se recomienda } \\
\text { la realización del «D test» para } \\
\text { descartar resistencia inducible a la } \\
\text { clindamicina, que desaconsejaría } \\
\text { su uso en casos positivos }\end{array}$ \\
\hline
\end{tabular}

$\mathrm{CMI}=$ concentración mínima inhibitoria; $\mathrm{EG}$ = edad gestacional, EMA = European Medicines Agency; $E \mathrm{EN}$ = edad postnatal; $F \mathrm{FQ}$ = fibrosis quística; IV = intravenoso; $\mathrm{LCR}$ = líquido cefalorraquídeo, $\mathrm{RC}$ = resistente a carbapenems; $\mathrm{UI}=$ unidades internacionales; $\mathrm{VO}$ = vía oral.

(1) La dosis específica en muchas ocasiones es off-label, por lo que conviene su uso con precaución. 


\begin{tabular}{|c|c|c|c|}
\hline Antibiótico & Dosis estándar & Dosis específica (1) & Comentarios \\
\hline Meropenem (IV) & $\begin{array}{l}\geq 1 \mathrm{~m}: 20 \text { mg/kg/8 horas (máx.: } 2 \\
\text { g/dosis) } \\
<1 \mathrm{~m}: \text { consultar dosis según peso, } \\
\text { EG y EPN }\end{array}$ & $\begin{array}{l}\text { En meningitis: } 40 \mathrm{mg} / \mathrm{kg} / 8 \text { horas } \\
\text { En infecciones por BGN RC (CMI } \\
\text { meropenem } \leq 8 \mathrm{mg} / \mathrm{L}) \text { : } \\
40 \mathrm{mg} / \mathrm{kg} / 8 \text { horas en perfusión de } \\
3 \text { horas }\end{array}$ & $\begin{array}{l}\text { Disminuye la concentración plas- } \\
\text { mática de ácido valproico (2) }\end{array}$ \\
\hline $\begin{array}{l}\text { Imipenem (imipenem- } \\
\text { cilastina) (IV) }\end{array}$ & $\begin{array}{l}\geq 3 \mathrm{~m}: 15-25 \mathrm{mg} / \mathrm{kg} / 6 \text { horas } \\
<3 \mathrm{~m} \text { y } \geq 1.5 \mathrm{~kg} \text { de peso, edad: } \\
1-3 \mathrm{~m}: 25 \mathrm{mg} / \mathrm{kg} / 6 \text { horas } \\
<1 \mathrm{~m}: \text { consultar dosis según peso, } \\
\text { EG y EPN } \\
\text { Máx.: } 4 \mathrm{~g} / \text { día }\end{array}$ & $\begin{array}{l}\text { Utilizar la dosis más elevada en } \\
\text { infecciones graves y/o por } P \text {. } \\
\text { aeruginosa }\end{array}$ & $\begin{array}{l}\text { No utilizar en infecciones del SNC. } \\
\text { Disminuye la concentración plas- } \\
\text { mática de ácido valproico (2). } \\
\text { Off-label en }<12 \text { meses de edad }\end{array}$ \\
\hline Ertapenem (IV) & $\begin{array}{l}>12 \text { a: } 1 \mathrm{~g} / 24 \text { horas } \\
3 \mathrm{~m}-12 \mathrm{a:} 15 \mathrm{mg} / \mathrm{kg} / 12 \text { horas (máx.: } \\
500 \mathrm{mg} / \text { dosis) }\end{array}$ & $\begin{array}{l}\text { Considerar } 2 \mathrm{~g} / \text { día y administración } \\
\text { c/12 horas en }>12 \text { años en el trata- } \\
\text { miento combinado de BGN RC }\end{array}$ & $\begin{array}{l}\text { Disminuye la concentración plas- } \\
\text { mática de ácido valproico (2) } \\
\text { No tiene actividad frente a } P \text {. } \\
\text { aeruginosa } \\
\text { Utilizar en infecciones por BGN RC } \\
\text { sólo en aislamientos panresistentes } \\
\text { en combinación con meropenem } \\
\text { (escasa experiencia) }\end{array}$ \\
\hline Gentamicina (IV) & $\begin{array}{l}\geq 1 \mathrm{~m}: 5-7.5 \mathrm{mg} / \mathrm{kg} / 24 \text { horas } \\
<1 \mathrm{~m}: \text { consultar dosis según peso, } \\
\text { EG y EPN }\end{array}$ & $\begin{array}{l}\text { Considerar } 7.5-10 \mathrm{mg} / \mathrm{kg} / 24 \text { horas } \\
\text { en pacientes con shock, infeccio- } \\
\text { nes pulmonares y fibrosis quística }\end{array}$ & $\begin{array}{l}\text { Concentración plasmática objetivo: } \\
\text { pico } 6-12 \mu \mathrm{g} / \mathrm{mL} \text {, valle }<2 \mu \mathrm{g} / \mathrm{mL}\end{array}$ \\
\hline Amikacina (IV) & $\begin{array}{l}\geq 1 \mathrm{~m}: 15-22 \mathrm{mg} / \mathrm{kg} / 24 \text { horas } \\
<1 \mathrm{~m}: \text { consultar dosis según peso, } \\
\text { EG y EPN }\end{array}$ & $\begin{array}{l}\text { Considerar 22-30 mg/kg/día en } \\
\text { pacientes con shock, infecciones } \\
\text { pulmonares y fibrosis quística }\end{array}$ & $\begin{array}{l}\text { Concentración plasmática objetivo: } \\
\text { pico } 20-35 \mu \mathrm{g} / \mathrm{mL} \text {, valle }<5 \mu \mathrm{g} / \mathrm{mL}\end{array}$ \\
\hline $\begin{array}{l}\text { Colistina (colistimeta- } \\
\text { to de sodio) (3) (IV) }\end{array}$ & $\begin{array}{l}75,000-150,000 \text { Ul/kg/día c/8-12 } \\
\text { horas (máx.: 10,800,000 UI/día) }\end{array}$ & $\begin{array}{l}\text { Considerar } 150,000-250,000 \mathrm{UI} / \\
\text { kg/día en pacientes con shock, } \\
\text { infecciones pulmonares, ITU grave } \\
\text { y fibrosis quística }\end{array}$ & $\begin{array}{l}\text { Se recomienda una dosis de } \\
\text { carga de } 75,000-150,000 \mathrm{Ul} / \mathrm{kg} \text { en } \\
\text { pacientes críticamente graves }\end{array}$ \\
\hline Tigeciclina (IV) & $\begin{array}{l}\geq 12 \text { a: } 50 \mathrm{mg} / 12 \text { horas } 8-11 \text { a: } 1,2 \\
\mathrm{mg} / \mathrm{kg} / 12 \text { horas (máx. } 50 \mathrm{mg} / 12 \\
\text { horas) }\end{array}$ & $\begin{array}{l}\text { Considerar dosis de carga y dosis } \\
\text { mayores en infecciones pulmo- } \\
\text { nares, ITU grave, bacteriemias o } \\
\text { shock: } \geq 12 \text { a: } 100-200 \mathrm{mg} \text { dosis de } \\
\text { carga y después } 100 \mathrm{mg} / 12 \text { horas } \\
\text { 8-11 a: } 2-3 \mathrm{mg} / \mathrm{kg} \text { dosis de carga } \\
\text { (máx.: } 200 \mathrm{mg} \text { ) y después } 2 \mathrm{mg} / \\
\mathrm{kg} / 12 \mathrm{~h} \text { (máx.: } 100 \mathrm{mg} / 12 \text { horas) }\end{array}$ & $\begin{array}{l}\text { Limitado a niños } \geq 8 \text { años con } \\
\text { infecciones por bacterias multi- } \\
\text { rresistentes sin otras opciones } \\
\text { terapéuticas } \\
\text { Evitar su uso en bacteriemia o neu- } \\
\text { monía, salvo falta de alternativas }\end{array}$ \\
\hline Fosfomicina (IV) & $\begin{array}{l}>12 \text { a (> } 40 \mathrm{~kg}): 12-24 \text { g/día c/6-8 } \\
\text { horas } \\
1-12 \text { a }(10-40 \mathrm{~kg}): 200-400 \mathrm{mg} / \mathrm{kg} / \\
\text { día c/6-8 horas } \\
1-12 \mathrm{~m}(\leq 10 \mathrm{~kg}): 200-300 \mathrm{mg} / \mathrm{kg} / \\
\text { día c/8 horas } \\
<1 \mathrm{~m} \text { : consultar dosis según peso, } \\
\text { EG y EPN } \\
\text { Máx.: } 8 \text { g/dosis; } 24 \mathrm{~g} / \text { día }\end{array}$ & $\begin{array}{l}\text { Considerar la dosis más alta en in- } \\
\text { fecciones graves, especialmente en } \\
\text { las causadas por microorganismos } \\
\text { con sensibilidad moderada }\end{array}$ & $\begin{array}{l}\text { Monitorizar sodio sérico y balance } \\
\text { hídrico debido al aporte elevado de } \\
\text { sodio ( } 330 \mathrm{mg} \text { por cada gramo de } \\
\text { fosfomicina) }\end{array}$ \\
\hline Aztreonam (IV) & $\begin{array}{l}\geq 1 \mathrm{~m}: 120-150 \mathrm{mg} / \mathrm{kg} / \mathrm{día} \text { c/8 horas } \\
\text { (máx.: } 8 \mathrm{~g} / \mathrm{día} \text { ) } \\
<1 \mathrm{~m}: \text { consultar dosis según peso, } \\
\text { EG y EPN }\end{array}$ & & $\begin{array}{l}\text { No utilizar en monoterapia en infec- } \\
\text { ciones por patógenos productores } \\
\text { de AmpC o BLEE }\end{array}$ \\
\hline
\end{tabular}




\section{Continúa la Tabla 2: Dosis de los principales antibióticos utilizados en pediatría para}

el tratamiento de infecciones por bacilos gramnegativos resistentes.

\begin{tabular}{|c|c|c|c|}
\hline Antibiótico & Dosis estándar & Dosis específica (1) & Comentarios \\
\hline $\begin{array}{l}\text { Piperacilina-tazobac- } \\
\text { tam (IV) }\end{array}$ & $\begin{array}{l}>12 \text { a y/0 } \geq 40 \text { kg: piperacilina } 2-4 \\
\text { g/dosis c/6-8 horas } \\
1 \mathrm{~m}-12 \text { a y/0 < } 40 \mathrm{~kg}: \text { piperacilina } \\
350-400 \mathrm{mg} / \mathrm{kg} / \text { día c/6-8 horas } \\
<1 \mathrm{~m} \text { : consultar dosis según peso, } \\
\text { EG y EPN } \\
\text { Máx.: } 16 \text { g/día de piperacilina }\end{array}$ & $\begin{array}{l}\text { Considerar la dosis más alta y } \\
\text { administración c/6h en infecciones } \\
\text { graves o con inóculo alto, valorando } \\
\text { perfusión extendida en } 4 \text { horas }\end{array}$ & $\begin{array}{l}\text { En el tratamiento de infecciones } \\
\text { por enterobacterales productores } \\
\text { de BLEE con sensibilidad in vitro } \\
\text { documentada administrar la dosis } \\
\text { más alta c/ } 6 \text { horas en perfusión } \\
\text { extendida en } 4 \text { horas }\end{array}$ \\
\hline Cefepima (IV) & $\begin{array}{l}\geq 2 \mathrm{~m}: 50 \mathrm{mg} / \mathrm{kg} / \text { dosis c/8 horas } \\
\text { (máx.: } 2,000 \mathrm{mg} / \mathrm{dosis} \text { ) } \\
<2 \mathrm{~m} \text { (off-label): consultar dosis } \\
\text { según peso, EG y EPN }\end{array}$ & $\begin{array}{l}\text { Considerar perfusión extendida en } \\
4 \text { horas en infecciones graves } 0 \\
\text { con inóculo alto }\end{array}$ & Aprobado en $\geq 2$ meses \\
\hline $\begin{array}{l}\text { Ceftazidima-avibactam } \\
\text { (IV) }\end{array}$ & $\begin{array}{l}\text { Perfusión IV en } 2 \text { horas: } 6 \text { m- } 18 \text { a: } \\
\text { < } 40 \mathrm{~kg}: 50 \mathrm{mg} / \mathrm{kg} \text { de ceftazidima } \\
\text { c/8 horas; } \geq 40 \mathrm{~kg}: 2 \text { g de ceftazidi- } \\
\text { ma c/8 horas } \\
\text { 3-6 m: } 40 \mathrm{mg} / \mathrm{kg} \text { de ceftazidima c/8 } \\
\text { horas } \\
\text { Máx.: } 2 \mathrm{~g} \text { de ceftazidima c/8 h }\end{array}$ & $\begin{array}{l}\text { Considerar perfusión en } 3 \text { horas en } \\
\text { infecciones graves. }\end{array}$ & $\begin{array}{l}\text { Actualmente aprobado por la FDA } \\
\text { en }>3 \text { meses. No aprobado por la } \\
\text { EMA en }<18 \text { años }\end{array}$ \\
\hline $\begin{array}{l}\text { Ceftolozano-tazobac- } \\
\text { tam (IV) }\end{array}$ & $\begin{array}{l}\text { Perfusión IV en } 1 \text { hora: } \\
\text { < } 18 \text { a: } 20 \text { mg/kg de ceftolozano } \\
\text { c/8 h (máx. } 1 \mathrm{~g} \text { de ceftolozano c/8 } \\
\text { horas) }\end{array}$ & $\begin{array}{l}\text { Considerar aumentar la dosis en } \\
\text { infecciones pulmonares graves: } \\
40 \mathrm{mg} / \mathrm{kg} \text { de ceftolozano } \mathrm{c} / 8 \text { horas } \\
\text { (máx. } 2 \mathrm{~g} \text { de ceftolozano } \mathrm{c} / 8 \text { horas) }\end{array}$ & $\begin{array}{l}\text { Actualmente no aprobado en }<18 \\
\text { años. Dosis procedente de ensayos } \\
\text { clínicos de fase I y II en niños }\end{array}$ \\
\hline \multicolumn{4}{|c|}{ 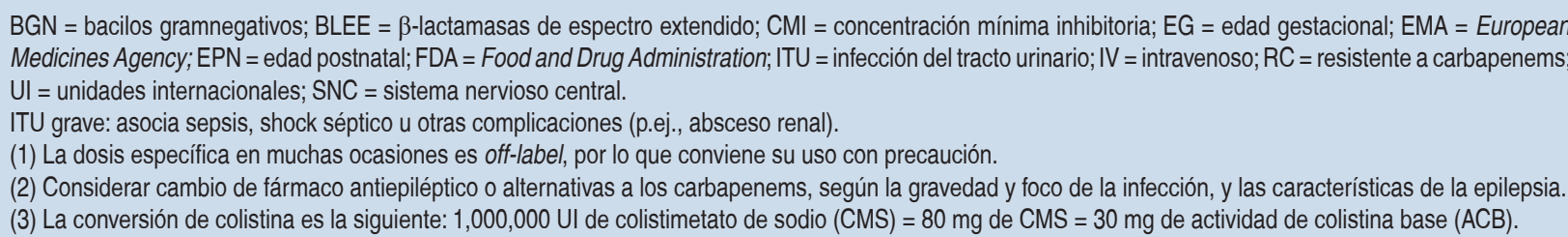 } \\
\hline
\end{tabular}

las cefalosporinas, lo que conlleva dificultades en su tratamiento. Además, también pueden adquirir resistencias (p.ej., a ampicilina y a vancomicina), ${ }^{9}$ disminuyendo todavía más las opciones terapéuticas.

E. faecalis es el microorganismo más frecuente y es casi uniformemente sensible a las aminopenicilinas, la ampicilina es el tratamiento de elección. E. faecium es el segundo en frecuencia y suele ser resistente a la ampicilina y sensible a la vancomicina.

Otro aspecto relevante de Enterococcus spp. es su resistencia intrínseca de bajo nivel a los aminoglucósidos por un transporte deficiente al interior de la bacteria. Sin embargo, cuando se asocian con otro antibiótico que actúa en la pared celular (p.ej., un $\beta$-lactámico o un glucopéptido), se produce un incremento de la penetración del aminoglucósido en el microorganismo, resultando en un efecto sinérgico bactericida, necesario para el tratamiento de infecciones graves (p.ej., endocarditis, meningitis y bacteriemia). Enterococcus spp. puede adquirir resistencia de alto nivel a aminoglucósidos, y en este caso no se produce sinergismo con glucopéptidos o $\beta$-lactámicos. Asimismo, se obtiene sinergismo bactericida con la asociación de ampicilina y ceftriaxona, pero solamente frente a $E$. faecalis.

\section{Epidemiología}

En aislamientos de $E$. faecium procedentes de hemocultivos en pacientes < 18 años en 2011-2012 en 12 países europeos, la prevalencia de resistencia a la vancomicina fue de $8.3 \% .^{10}$ No obstante, según los datos reportados por el ECDC, en los últimos años la 
resistencia a la vancomicina en España en muestras invasivas procedentes de todos los grupos etarios ha sido de $0.1-0.3 \%$ para $E$. faecalis y de $1.8-2.5 \%$ para $E$. faecium, presentando una tendencia estable.

\section{Tratamiento}

Siempre que el aislamiento sea sensible a la ampicilina, será el tratamiento de elección (Tabla 4). La alternativa son los glucopéptidos (vancomicina o teicoplanina). En el caso de infecciones graves, como endocarditis, meningitis o bacteriemia, se recomienda la combinación de dos antibióticos con el objetivo de alcanzar una sinergia bactericida. ${ }^{11}$ Las combinaciones más empleadas son ampicilina junto con gentamicina o ceftriaxona en $E$. faecalis y vancomicina con gentamicina en $E$. faecium.

Cuando se administran combinaciones con aminoglucósidos hay que evaluar previamente in vitro si existe resistencia de alto nivel mediante la determinación de la CMI. En el caso de resistencia de alto nivel (CMI de gentamicina $\geq 500 \mathrm{mg} / \mathrm{L}$ o de estreptomicina $\geq 2,000 \mathrm{mg} / \mathrm{L}$ ) habría que utilizar alternativas como ampicilina con ceftriaxona en el caso de $E$. faecalis. Cuando la cepa es resistente a la ampicilina y a los glucopéptidos, la daptomicina o el linezolid pueden ser una alternativa.

\section{INFECCIONES POR BACILOS GRAMNEGATIVOS}

\author{
Enterobacterales productores de \\ $\beta$-lactamasas de espectro extendido
}

Las $\beta$-lactamasas de espectro extendido (BLEE) son enzimas que tienen capacidad de hidrolizar y causar resistencia o sensibilidad disminuida a penicilinas, oximinocefalosporinas (cefotaxima, ceftriaxona, ceftazidima y cefepima) y a monobactámicos (aztreonam), pero no a cefamicinas (cefoxitina) ni a carbapenems. Generalmente son inhibidas in vitro por varios inhibidores de las $\beta$-lactamasas (p.ej., ácido clavulánico, tazobactam o avibactam).

Las cepas productoras de BLEE por lo regular contienen otros genes que confieren resistencia a los aminoglucósidos (principalmente gentamicina y con menos frecuencia a amikacina), al cotrimoxazol o a las fluoroquinolonas.

\section{Epidemiología}

En los últimos años se ha evidenciado un incremento notable en la prevalencia de enterobacterales productores de BLEE, tanto a nivel hospitalario como comunitario. ${ }^{12}$ Entre los factores de riesgo asociados

Tabla 3: Tratamiento de las infecciones producidas por S. aureus resistente a meticilina.

\begin{tabular}{ccc}
\hline Infección & Tratamiento de elección & Alternativa \\
\hline IPPB (1) & Clindamicina (2) o cotrimoxazol & $\begin{array}{c}\text { Linezolid, vancomicina, teicoplanina, } \\
\text { ceftarolina o daptomicina }\end{array}$ \\
Neumonía (3) & Clindamicina (2), vancomicina o linezolid & Ceftarolina, teicoplanina o cotrimoxazol \\
Bacteriemia y endocarditis (4)(5) & Vancomicina o daptomicina & Teicoplanina, linezolid o ceftarolina \\
IOA (6) & Clindamicina o cotrimoxazol (2) & Vancomicina, linezolid o levofloxacino \\
SNC & Vancomicina (7) & Linezolid \\
\hline
\end{tabular}

IPPB = infecciones de piel y partes blandas; IOA = infección osteoarticular; SNC = sistema nervioso central. Considerar el orden de aparición en la tabla como el orden de elección.

(1) En el caso de IPPB graves priorizar linezolid o clindamicina.

(2) Cuando se indique tratamiento oral, no existe solución de clindamicina comercializada en algunos países, por lo que cotrimoxazol constituye una posible alternativa.

(3) En el caso de neumonía grave priorizar vancomicina + clindamicina o linezolid.

(4) En el caso de bacteriemia persistente: si tratamiento previo con vancomicina, cambiar a daptomicina. Si tratamiento con daptomicina, asociar ceftarolina 0 fosfomicina. En el caso de presencia de material protésico valorar asociar rifampicina. Se define bacteriemia persistente como la persistencia de hemocultivos positivos tras 72 horas o más del inicio de un tratamiento adecuado (incluyendo la retirada de catéter) o la aparición de tromboembolismos sépticos o metástasis a distancia tras 72 horas de antibioterapia.

(5) En el caso de endocarditis sobre válvula protésica se recomienda: (vancomicina o daptomicina) + rifampicina + gentamicina.

(6) En el caso de infecciones de prótesis osteoarticular con retención del material de osteosíntesis asociar la rifampicina. Algunos autores recomiendan iniciarla en una segunda fase dirigida al tratamiento del biofilm, tras siete días de tratamiento. ${ }^{7}$

(7) Asociar la rifampicina a la vancomicina en las infecciones con mantenimiento de material extraño (p.ej., válvula de derivación ventricular) y considerarlo en el resto de casos. La rifampicina disminuye la concentración plasmática de linezolid 30\%, por lo que esta combinación no es recomendable. 


\section{Tabla 4: Tratamiento antimicrobiano de infecciones por Enterococcus spp.}

\begin{tabular}{|c|c|c|c|c|}
\hline \multirow[t]{3}{*}{ Antibiótico principal } & De elección (1) & \multicolumn{3}{|l|}{ Ampicilina (2) } \\
\hline & Si R a ampicilina & \multicolumn{3}{|l|}{$\begin{array}{l}\text { Vancomicina o } \\
\text { teicoplanina (3) }\end{array}$} \\
\hline & $\begin{array}{l}\text { Si R a ampicilina y } \\
\text { glucopéptidos }\end{array}$ & \multicolumn{3}{|l|}{$\begin{array}{l}\text { Daptomicina (4) } 0 \\
\text { linezolid (5) }\end{array}$} \\
\hline \multirow[t]{3}{*}{ Combinación } & Infecciones leves & No precisa & & \\
\hline & $\begin{array}{l}\text { Infecciones graves } \\
\text { (endocarditis, menin- }\end{array}$ & $\begin{array}{l}\text { Ausencia de RAN } \\
\text { a aminoglucósidos }\end{array}$ & \multicolumn{2}{|l|}{$\begin{array}{l}\text { Gentamicina (junto a } \\
\text { ampicilina o vancomicina) }\end{array}$} \\
\hline & & $\begin{array}{l}\text { RAN a } \\
\text { aminoglucósidos }\end{array}$ & $\begin{array}{l}\text { Si S a ampicilina } \\
\text { Si R a ampicilina (6) }\end{array}$ & $\begin{array}{l}\text { Ampicilina + ceftriaxona (sólo para a } \\
\text { E. faecalis) } \\
\text { Daptomicina (4) + (ceftarolina, ampici- } \\
\text { lina (3) o fosfomicina), vancomicina }+ \\
\text { rifampicina o linezolid } \pm \text { (fosfomicina } 0 \\
\text { fluoroquinolonas) }\end{array}$ \\
\hline \multicolumn{5}{|c|}{$\begin{array}{l}\mathrm{S}=\text { sensible; } \mathrm{R}=\text { resistente; } \mathrm{RAN}=\text { resistencia de alto nivel (CMI } \geq 500 \mathrm{mg} / \mathrm{L} \text { de gentamicina). } \\
\text { (1) Si infección urinaria: puede tratarse con amoxicilina, fosfomicina, nitrofurantoína o fluoroquinolonas. } \\
\text { (2) E. faecium suele ser resistente a la ampicilina, por lo que no se recomienda su uso de forma empírica frente a esta especie. } \\
\text { (3) En el caso de CMI de ampicilina } \leq 64 \mathrm{mg} / \mathrm{L} \text { podría valorarse el tratamiento con dosis altas de ampicilina. Considerar tratamiento combinado en estos casos. } \\
\text { (4) La daptomicina, debido a su inactivación por el surfactante pulmonar, no se recomienda en el tratamiento de infecciones respiratorias. Además, presenta baja } \\
\text { penetración en el sistema nervioso central. Utilizar dosis altas (Tabla 1) en infecciones graves. } \\
\text { (5) En el tratamiento de endocarditis actualmente existe mayor experiencia con daptomicina que con linezolid. } \\
\text { (6) La experiencia con las combinaciones propuestas es escasa, por lo que se recomienda siempre consultar con expertos. }\end{array}$} \\
\hline
\end{tabular}

a infecciones por bacterias productoras de BLEE destacan el ingreso hospitalario previo, cirugía o antibioterapia recientes, principalmente cefalosporinas de tercera generación, fluoroquinolonas y carbapenems, y la presencia de patología crónica. ${ }^{13}$

\section{Tratamiento}

La elección del tratamiento antibiótico está basada en el foco y gravedad de la infección, y el estado inmunológico del paciente ${ }^{3}$ (Tabla 5). Tradicionalmente, los carbapenems han sido los antibióticos de elección en infecciones con este perfil de resistencia. Sin embargo, el incremento de la prevalencia de microorganismos resistentes a carbapenems ha remarcado la necesidad de definir antibióticos «ahorradores» de carbapenems que aseguren una efectividad similar sin favorecer la selección de cepas resistentes.

Aunque las bacterias productoras de BLEE presentan con frecuencia sensibilidad in vitro a la piperacilina-tazobactam, se han descrito fracasos terapéuticos, sobre todo en presencia de infecciones con un alto inóculo (p.ej., neumonía, abscesos no drenados, etcétera). Estudios observacionales han demostrado la eficacia de la piperacilina-tazobactam, principalmente en infecciones con bajo inóculo, infecciones no graves y mediante la administración en perfusión extendida. ${ }^{3}$

El uso de otros antibióticos (p.ej., aminoglucósidos, cotrimoxazol, amoxicilina-clavulánico, fosfomicina, cefoxitina o fluoroquinolonas) puede ser una opción en el caso de ITU no grave, y como paso secuencial de vía intravenosa a vía oral tras el control inicial del foco infeccioso. En enterobacterales productores de BLEE, el ertapenem también es una buena alternativa al meropenem en algunos casos, pues permite disminuir la presión antibiótica sobre Pseudomonas aeruginosa, además de las ventajas que conlleva su administración más espaciada.

\section{Enterobacterales productores de AmpC}

Las $\beta$-lactamasas de tipo AmpC pueden ser cromosómicas (intrínsecas) o plasmídicas (adquiridas). Enterobacter spp., Citrobacter freundii complex, Serratia spp., Providencia spp., Morganella morganii y la especie recientemente denominada Klebsiella aerogenes (antes Enterobacter aerogenes) presentan constitutivamente el gen para la producción de AmpC, y confieren resistencia intrínseca a la ampicilina, a la amoxicilina-clavulánico y a las cefalosporinas 
de primera y segunda generación, pero permanecen sensibles in vitro a las cefalosporinas de tercera y cuarta generación, así como a la piperacilina.

En el caso de la exposición a $\beta$-lactámicos, principalmente cefalosporinas y penicilinas, se puede producir desrepresión del gen e hiperproducción de la enzima, confiriendo el fenotipo característico AmpC con resistencia a todos los $\beta$-lactámicos (salvo cefepima, carbapenémicos y las combinaciones con los nuevos inhibidores de las $\beta$-lactamasas como ceftazidima-avibactam). Enterobacter spp. presenta el mayor riesgo de inducción de las AmpC. ${ }^{14}$

\section{Tratamiento}

En el caso de infecciones graves por microorganismos que producen AmpC, debido a la falta de evidencia suficiente, el uso de carbapenems resulta la opción más recomendable (Tabla 6). ${ }^{14}$ Cuando pueda utilizarse, el tratamiento dirigido con ertapenem podría ayudar a reducir la presión antibiótica frente a $P$. aeruginosa. Algunos $\beta$-lactámicos no carbapenems, como la cefepima o la piperacilinatazobactam, se consideran inductores débiles, por lo que pueden ser una alternativa en algunas circunstancias mediante el uso de dosis alta y perfusión extendida. ${ }^{15}$ Se recomienda evitar el uso de piperacilina-tazobactam en infecciones con inóculo alto o con aislamientos con CMI de piperacilina-tazobactam $>8 \mathrm{mg} / \mathrm{L}$, y de cefepima en aislamientos con CMI de cefepima $>1 \mathrm{mg} / \mathrm{L} .{ }^{16} \mathrm{La}$ cefepima generalmente no se hidroliza por las $\mathrm{AmpC}$, por lo que es una excelente opción. En el tratamiento de infecciones del tracto urinario (ITU) no graves podrían plantearse varias alternativas según la sensibilidad del aislamiento en el antibiograma (p.ej., aminoglucósidos, cotrimoxazol, fluoroquinolonas, etcétera).

\section{Bacilos gramnegativos resistentes a carbapenems}

\section{Epidemiología}

El desarrollo de resistencia a los carbapenems constituye uno de los escenarios más preocupantes dentro de las resistencias a antimicrobianos al perderse la actividad de uno de los grupos con mayor espectro. En el caso de los enterobacterales, esta resistencia generalmente se debe a la producción de carbapenemasas, mientras que en el caso de $P$. aeruginosa suele deberse a mecanismos no enzimáticos (p.ej., inactivación o represión de las porinas e hiperproducción de bombas de expulsión activa).

Estudios recientes realizados en Estados Unidos han demostrado un incremento en la prevalencia de infecciones por bacilos gramnegativos (BGN) RC en niños: de $0 \%$ en $1999-2000$ a $0.47 \%$ en $2010-2011$

Tabla 5: Tratamiento de las infecciones por enterobacterales productores de $\beta$-lactamasas de espectro extendido.

Características de la infección

ITU no grave (1)

Infecciones no graves (2)

Infecciones graves (sepsis grave o shock séptico) o inmunosupresión grave

\section{Antibióticos}

Aminoglucósidos, cotrimoxazol, amoxicilina-clavulánico, fosfomicina, cefoxitina o fluoroquinolonas Foco de inóculo bajo-intermedio (3)

Foco de inóculo alto (4) Meropenem o imipenem (7)
Piperacilina-tazobactam (dosis alta en perfusión extendida) (5) o ertapenem Meropenem, imipenem o ertapenem (6)

ITU = infecciones del tracto urinario.

(1) ITU no grave: no asocia sepsis, shock séptico ni otras complicaciones (p.ej., absceso renal).

(2) Considerar paso secuencial a vía oral, según el foco de la infección, tras objetivar mejoría clínica y analítica, con cualquiera de las opciones incluidas en el tratamiento de ITU no grave.

(3) Foco de inóculo bajo-intermedio: infección de catéter vascular con retirada del mismo, infecciones cutáneas (drenadas en el caso de presentar absceso), infecciones profundas (p.ej., intraabdominal) correctamente drenadas o ITUs.

(4) Foco de inóculo alto: neumonía, endocarditis, infecciones del sistema nervioso central e infecciones profundas inadecuadamente drenadas.

(5) Considerar su uso en el caso de CMI de piperacilina-tazobactam $\leq 8 \mathrm{mg} / \mathrm{L}$.

(6) En el caso de neumonías, si CMI de piperacilina-tazobactam < $4 \mathrm{mg} / \mathrm{L}$, podría utilizarse piperacilina-tazobactam siguiendo la indicación de un experto, con una estrecha vigilancia.

(7) Podría valorarse desescalar a piperacilina-tazobactam o ertapenem una vez controlada la infección.

Adaptada con permiso de Gutiérrez-Gutiérrez B. ${ }^{8}$ 
Tabla 6: Tratamiento de infecciones por microorganismos con producción constitutiva o adquirida de AmpC.

Características de la infección

ITU no grave (1)(2)

Infecciones no graves (3)

Infecciones graves (sepsis grave o shock

séptico) 0 inmunosupresión grave
Antibióticos

Aminoglucósidos, cotrimoxazol, fosfomicina o fluoroquinolonas

Foco de inóculo intermedio-bajo (5)(6) Cefepima (4) o piperacilina-tazobactam

Foco de inóculo alto (5)(7)

Meropenem o imipenem (7)
Meropenem, imipenem, ertapenem 0 cefepima (4)(8)

ITU = infección del tracto urinario

(1) ITU no grave: no asocia sepsis, shock séptico ni otras complicaciones (p.ej., absceso renal).

(2) En el caso de ITU no grave por Serratia spp., Providencia spp. o Morganella morganii podría tratarse con cefotaxima o ceftriaxona si se confirma la sensibilidad a éstos, con una estrecha vigilancia.

(3) Ver clasificación de foco de la infección según el inóculo en el pie de la Tabla 5.

(4) Utilizar únicamente en aislamientos con CMI de cefepime $\leq 1 \mathrm{mg} / \mathrm{L}$. En infecciones con foco de inóculo intermedio 0 alto administrar cada ocho horas en perfusión extendida (en cuatro horas).

(5) Considerar paso secuencial a vía oral, según el foco de la infección, tras objetivar mejoría clínica y analítica, con cualquiera de las opciones incluidas en el tratamiento de ITU no grave.

(6) Podría utilizarse como alternativa fluoroquinolonas.

(7) Considerar desescalar a cefepima, piperacilina-tazobactam o fluoroquinolonas una vez controlada la infección. Consultar con experto previamente.

(8) Evitar cefepima en abscesos o infecciones profundas inadecuadamente drenados, endocarditis e infecciones del sistema nervioso central.

en el caso de enterobacterales, ${ }^{17}$ y de $9.4 \%$ en 1999 a $20 \%$ en 2012 en $P$. aeruginosa. ${ }^{18}$

En la mayoría de los países, las infecciones por estas cepas suceden principalmente en población adulta. En niños se ha descrito mayoritariamente en relación con brotes hospitalarios, con la excepción de países con una alta prevalencia. Las infecciones por estos microorganismos presentan mayor mortalidad $^{19} \mathrm{y}$ comparten factores de riesgo similares a los observados en los BGN productores de BLEE. ${ }^{20}$

\section{Tratamiento}

Actualmente, se recomienda tratamiento combinado con dos o más antibióticos activos frente al microorganismo aislado en la mayoría de los casos (Figura 1). El estudio INCREMENT, realizado en adultos, sugiere el uso de monoterapia en infecciones con menor riesgo de gravedad. ${ }^{21}$ Sin embargo, debido a múltiples aspectos, como las escalas utilizadas o el mayor desconocimiento sobre la farmacocinética/ farmacodinamia de muchos antibióticos en niños, su extrapolación al paciente pediátrico resulta complicada. En la Tabla 7 se presentan las posibles combinaciones según el foco de la infección. En infecciones no graves (por ejemplo, ITU sin criterios de gravedad) podría valorarse el uso de monoterapias.

El uso de pautas optimizadas de meropenem (a doble dosis en perfusión extendida) posibilita alcan- zar los objetivos farmacodinámicos en el caso de aislamientos con una $\mathrm{CMI}$ de meropenem $\leq 8 \mathrm{mg} / \mathrm{L},{ }^{22}$ y ha demostrado una eficacia adecuada en estudios observacionales. ${ }^{23,24}$ Por ello, consideramos que es actualmente el tratamiento de elección en combinación con un segundo antibiótico activo in vitro. ${ }^{2,25} \mathrm{En}$ el caso de aislamientos con CMI de meropenem $>8$ $\mathrm{mg} / \mathrm{L}$, la alternativa de elección sería un $\beta$-lactámico activo basado en los resultados del antibiograma interpretado. En el caso de no disponer de alguno que preserve actividad frente al aislamiento, otras alternativas podrían ser colistina, aminoglucósidos, fosfomicina, fluoroquinolonas o tigeciclina.

Las nuevas combinaciones de $\beta$-lactámicosinhibidores de las $\beta$-lactamasas (cefazidima-avibactam y ceftolozano-tazobactam) actualmente carecen de aprobación en edad pediátrica. Sin embargo, estudios en fase II han demostrado su seguridad y eficacia en niños, habiendo sido la ceftazidimaavibactam recientemente aprobada por la FDA (Food and Drug Administration) en mayores de tres meses de edad. Por tanto, su uso off-label podría contemplarse en el caso de aislamientos con CMI de meropenem $>8 \mathrm{mg} / \mathrm{L}$ y demostrada sensibilidad al antibiótico, priorizándose su uso en el caso de infecciones graves.

En el caso de aislamientos extremadamente resistentes, con escasas opciones terapéuticas, se recomienda el estudio de posibles sinergias por 
el laboratorio de microbiología (p.ej., aztreonamavibactam, meropenem-ertapenem, meropenemfosfomicina. ${ }^{2}$ La combinación de aztreonam con avibactam (actualmente con ceftazidima-avibactam, al no estar comercializada todavía la combinación) ha demostrado su eficacia en cepas productoras de MBL resistentes al aztreonam. Por ello, podría ser una alternativa frente a este tipo de carbapenemasas en el caso de no disponer de otras opciones terapéuticas. $^{2}$

\section{CONCLUSIÓN}

En los últimos años se ha evidenciado un incremento progresivo en la incidencia de infecciones por BMR, su tratamiento supone un reto importante. Para hacer frente a este problema, es fundamental establecer programas de optimización en el uso de antimicro- bianos, realizar una vigilancia epidemiológica activa y desarrollar una adecuada política de control de infecciones. La escasez de opciones terapéuticas incrementadas en el paciente pediátrico por la falta de ensayos clínicos dificulta la elección de un tratamiento efectivo. El desarrollo de nuevos antibióticos va a permitir mejorar el pronóstico asociado a estas infecciones. Sin embargo, el uso prudente de los mismos es un aspecto primordial para preservar su actividad.

\section{AGRADECIMIENTOS}

Los autores agradecen la revisión del manuscrito a Natalia Mendoza Palomar (Unitat de Patologia Infecciosa i Immunodeficiències de Pediatria, Hospital Universitari Vall d'Hebron), M. Nieves Larrosa Escartín (Servei de Microbiologia, Hospital Universitari Vall

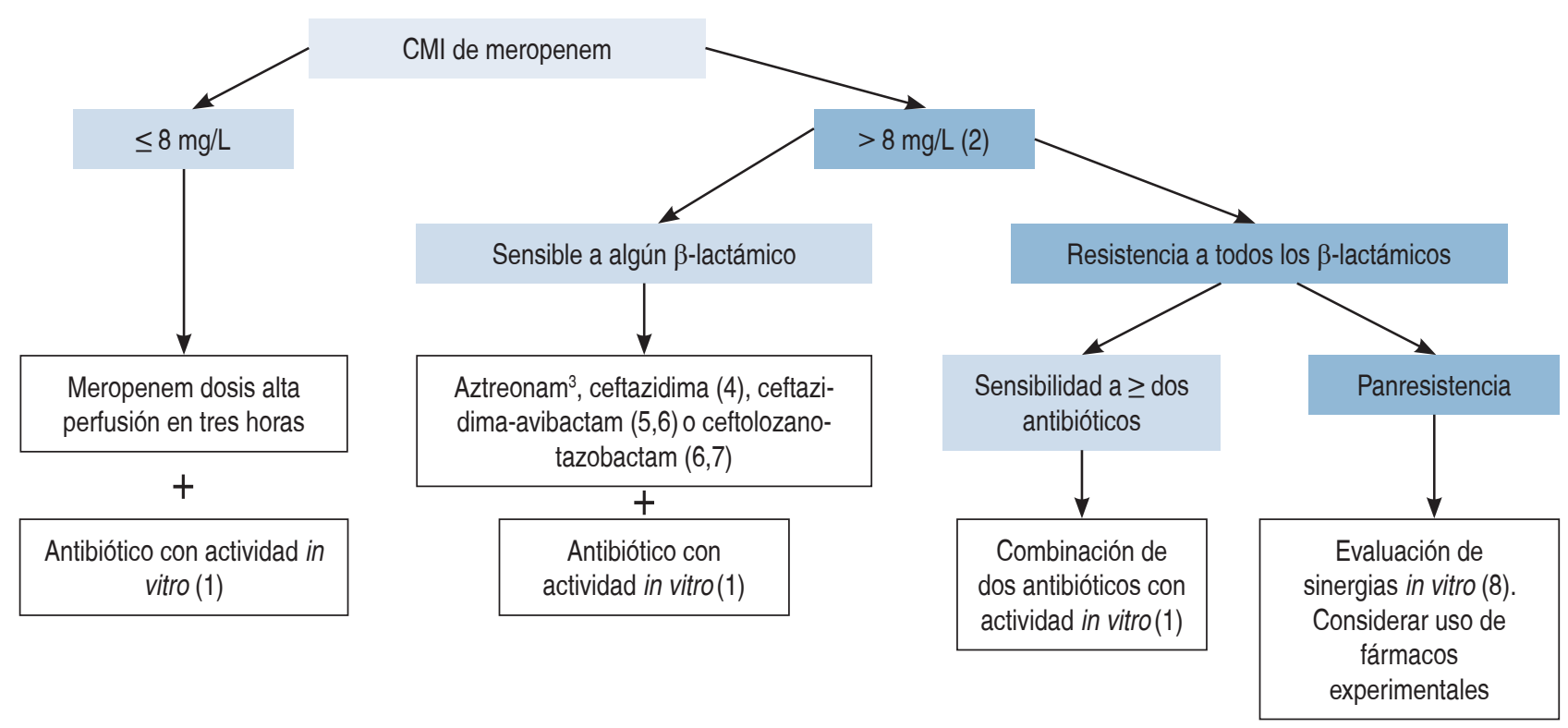

Figura 1: Tratamiento de las infecciones por bacilos gramnegativos resistentes a carbapenems en niños.

(1) Posibles antibióticos: aminoglucósido, colistina, fosfomicina, fluoroquinolona o tigeciclina. Considerar el foco de la infección y la sensibilidad del aislamiento en la elección (Tabla 7).

(2) Considerar si CMI de meropenem $>4 \mathrm{mg} / \mathrm{L}$, especialmente en infecciones graves.

(3) El aztreonam puede utilizarse en infecciones por BGN productores de MBL (VIM, NDM o IMP) u OXA-48, si no se detecta coproducción de BLEE o AmpC; en el caso de detectarse, considerar su utilización en combinación con ceftazidima-avibactam si se demuestra sinergia in vitro.

(4) La ceftazidima puede utilizarse en infecciones por BGN productores de OXA-48 si no se detecta coproducción de BLEE o AmpC.

(5) La ceftazidima-avibactam puede utilizarse en infecciones por BGN RC no mediadas por MBL.

(6) Actualmente, la ceftazidima-avibactam o el ceftolozano-tazobactam, debido a su uso off-label en niños, debería ser limitado a infecciones graves en las que el microorganismo aislado no presente sensibilidad in vitro a otros $\beta$-lactámicos, o a infecciones por microorganismos panresistentes. Datos procedentes de adultos sugieren que el tratamiento combinado no es necesario cuando se usan los nuevos $\beta$-lactámicos-inhibidores de las $\beta$-lactamasas.

(7) El ceftolozano-tazobactam puede utilizarse en infecciones por $P$. aeruginosa $\mathrm{RC}$ no mediada por carbapenemasas.

(8) Posibles sinergias in vitro documentadas, pero con poca experiencia clínica: ceftazidima-avibactam + aztreonam, meropenem + ertapenem, meropenem + fosfomicina, ceftazidima-avibactam + fosfomicina.

Adaptado de: Hsu AJ. ${ }^{25}$ 
Tabla 7: Antibióticos en infecciones por bacilos gramnegativos resistentes a carbapenems según el foco de la infección.

\begin{tabular}{|c|c|c|c|c|}
\hline Foco de infección & Antibiótico principal & $\begin{array}{l}\text { Combinación de } \\
\text { elección }\end{array}$ & Combinación alternativa & Otras alternativas (1) \\
\hline $\begin{array}{c}\text { Respiratorio } \\
\text { Urinario } \\
\text { BRCV } \\
\text { Intraabdominal (4) }\end{array}$ & $\begin{array}{c}\beta \text {-lactámico } \\
\text { Aminoglucósido (3) } \\
\beta \text {-lactámico } \\
\beta \text {-lactámico }\end{array}$ & $\begin{array}{l}\text { Fluoroquinolona } \\
\beta \text {-lactámico } \\
\text { Colistina } \\
\text { Tigeciclina }\end{array}$ & $\begin{array}{l}\text { Colistina (2) o aminoglucósido (2) } \\
\text { Fosfomicina o fluoroquinolona } \\
\text { Fosfomicina o aminoglucósido } \\
\text { Colistina }\end{array}$ & $\begin{array}{l}\text { Fosfomicina o tigeciclina (2) } \\
\text { Colistina (2) o tigeciclina (2) } \\
\text { Fluoroquinolona o tigeciclina (2) } \\
\text { Aminoglucósido, fluoroquino- } \\
\text { Iona o fosfomicina }\end{array}$ \\
\hline \multicolumn{5}{|c|}{$\begin{array}{l}\text { BRCV = bacteriemia relacionada con el catéter vascular. } \\
\text { Se utilizarán los antibióticos en la siguiente tabla siguiendo el algoritmo de la Figura 1, teniendo en cuenta la sensibilidad del aislamiento in vitro. Se recomienda } \\
\text { siempre consultar con un experto. } \\
\text { (1) Valorar su uso en caso de no tener disponibles las alternativas de elección. } \\
\text { (2) En el caso de utilizar en estos focos, se recomienda optimizar su posología (Tabla 2). } \\
\text { (3) En el caso de ITU no grave (no asocia sepsis, shock séptico ni otras complicaciones), considerar tratamiento en monoterapia. } \\
\text { (4) En el tratamiento de infecciones intraabdominales, con la excepción de tigeciclina o meropenem, el resto de los antibióticos deberán combinarse con un } \\
\text { antibiótico con actividad anaerobicida (p.ej., metronidazol). }\end{array}$} \\
\hline
\end{tabular}

d'Hebron), Silvia Manrique Rodríguez (Servicio de Farmacia, Hospital General Universitario Gregorio Marañón), Cecilia M. Fernández-Llamazares (Servicio de Farmacia, Hospital General Universitario Gregorio Marañón), Montserrat Giménez Pérez (Servicio de Microbiologia, Hospital Universitario Germans Trias i Pujol) y a Emilio Cendejas (Servicio de Microbiología y Parasitología, Hospital Universitario La Paz).

\section{REFERENCIAS}

1. Cassini A, Högberg LD, Plachouras D, Quattrocchi A, Hoxha A, Simonsen GS et al. Attributable deaths and disabilityadjusted life-years caused by infections with antibioticresistant bacteria in the EU and the European Economic Area in 2015: a population-level modelling analysis. Lancet Infect Dis. 2019; 19: 56-66.

2. Rodríguez-Baño J, Gutiérrez-Gutiérrez B, Machuca I, Pascual A. Treatment of infections caused by ExtendedSpectrum-Beta-Lactamase-, Ampc-, and Carbapenemaseproducing Enterobacteriaceae. Clin Microbiol Rev. 2018; 31 (2). pii: e00079-17.

3. Gutierrez-Gutiérrez B, Rodríguez-Baño J. Current options for the treatment of infections due to extended-spectrum beta-lactamase-producing Enterobacteriaceae in different groups of patients. Clin Microbiol Infect. 2019; 25 (8): 932942.

4. McMullan BJ, Andresen D, Blyth CC, Avent ML, Bowen $\mathrm{AC}$, Britton PN et al. Antibiotic duration and timing of the switch from intravenous to oral route for bacterial infections in children: systematic review and guidelines. Lancet Infect Dis. 2016; 16: e139-e152.

5. Turner NA, Sharma-Kuinkel BK, Maskarinec SA, Eichenberger EM, Shah PP, Carugati M et al. Methicillinresistant Staphylococcus aureus: an overview of basic and clinical research. Nat Rev Microbiol. 2019; 17: 203-218.
6. Ara Montojo MF, Aguilera-Alonso D, Del Rosal T, Sanz Santaeufemia FJ, Berzosa A, Soto Sánchez ATB. High rate of methillin resistance among Staphylococcus aureus isolates from Spanish children with community-acquired pneumoniale. In: 37th Annual Meeting of the European Society for Paediatric Infectious Diseases. 2019.

7. Ariza J, Cobo J, Baraia-Etxaburu J, Benito N, Bori G, Cabo $\mathrm{J}$ et al. Executive summary of management of prosthetic joint infections. Clinical practice guidelines by the Spanish Society of Infectious Diseases and Clinical Microbiology (SEIMC). Enferm Infecc Microbiol Clin. 2017; 35: 189-195.

8. Cosimi RA, Beik N, Kubiak DW, Johnson JA. Ceftaroline for severe methicillin-resistant Staphylococcus aureus infections: a systematic review. Open Forum Infect Dis. 2017; 4: ofx084.

9. Cercenado E. Enterococcus: phenotype and genotype resistance and epidemiology in Spain. Enferm Infecc Microbiol Clin. 2011; 29: 59-65.

10. Bielicki JA, Lundin R, Sharland M, ARPEC Project. Antibiotic Resistance Prevalence in Routine Bloodstream Isolates from Children's Hospitals Varies Substantially from Adult Surveillance Data in Europe. Pediatr Infect Dis J. 2015; 34: 734-741.

11. Yim J, Smith JR, Rybak MJ. Role of combination antimicrobial therapy for vancomycin-resistant enterococcus faecium infections: review of the current evidence. Pharmacother J Hum Pharmacol Drug Ther. 2017; 37: 579592.

12. Flokas ME, Karanika S, Alevizakos M, Mylonakis E. Prevalence of ESBL-Producing Enterobacteriaceae in pediatric bloodstream infections: a systematic review and meta-analysis. PLoS One. 2017; 12: e0171216.

13. Yanhong Jessika Hu, Anju Ogyu, Benjamin J Cowling, Keiji Fukuda HHP. Available evidence of antibiotic resistance from extended-spectrum $\beta$-lactamase-producing Enterobacteriaceae in paediatric patients in 20 countries: a systematic review and meta-analysis. Bull World Health Organ. 2019; 1: 486-501B.

14. Tamma PD, Doi Y, Bonomo RA, Johnson JK, Simner PJ; Antibacterial Resistance Leadership Group. A primer 
on AmpC $\beta$-Lactamases: necessary knowledge for an increasingly multidrug-resistant world. Clin Infect Dis. 2019; 69 (8): 1446-1455.

15. Cheng MP, Lee RS, Cheng AP, De L'étoile-Morel S, Demir K, Yansouni CP et al. Beta-Lactam/beta-lactamase inhibitor therapy for potential AmpC-Producing organisms: a systematic review and meta-analysis. Open Forum Infect Dis. 2019; 6 (7). pii: ofz248.

16. Gudiol C, Aguilar-Guisado M, Azanza JR, Candel FJ, Cantón $\mathrm{R}$, Carratalà $\mathrm{J}$ et al. Executive summary of the consensus document of the Spanish Society of Infectious Diseases and Clinical Microbiology (SEIMC), the Spanish Network for Research in Infectious Diseases (REIPI) and the Spanish Society of Haematology and Haemotherapy (SEHH) on the management of febrile neutropenia in patients with hematological malignancies. Enferm Infecc Microbiol Clin. 2019; pii: S0213-005X(19)30130-2.

17. Logan LK, Renschler JP, Gandra S, Weinstein RA, Laxminarayan R; Centers for Disease Control; Prevention Epicenters Program. Carbapenem-resistant enterobacteriaceae in children, United States, 1999-2012. Emerg Infect Dis. 2015; 21: 2014-2021.

18. Logan LK, Gandra S, Mandal S, Klein EY, Levinson J, Weinstein RA et al. Multidrug- and carbapenem-resistant Pseudomonas aeruginosa in Children, United States, 19992012. J Pediatric Infect Dis Soc. 2016; 6: 352-359.

19. Chiotos K, Tamma PD, Flett KB, Karandikar MV, Nemati K, Bilker WB et al. Increased 30-day mortality associated with carbapenem-resistant enterobacteriaceae in children. Open Forum Infect Dis. 2018; 5: ofy222.

20. Chiotos K, Tamma PD, Flett KB, Naumann M, Karandikar MV, Bilker WB et al. Multicenter study of the risk factors for colonization or infection with carbapenem-resistant enterobacteriaceae in children. Antimicrob Agents Chemother. 2017 Dec; 61 (12): e01440-01417.

21. Gutiérrez-Gutiérrez $B$, Salamanca $E$, de Cueto $M$, Hsueh PR, Viale P, Paño-Pardo JR et al. Effect of appropriate combination therapy on mortality of patients with bloodstream infections due to carbapenemase-producing Enterobacteriaceae (INCREMENT): a retrospective cohort study. Lancet Infect Dis. 2017; 17: 726-734.

22. Cies JJ, Moore WS, Enache A, Chopra A, Chopra A. Population pharmacokinetics and pharmacodynamic target attainment of meropenem in critically ill young children. $J$ Pediatr Pharmacol Ther. 2017; 22: 276-285.

23. Daikos GL, Markogiannakis A. Carbapenemase-producing Klebsiella pneumoniae: (when) might we still consider treating with carbapenems? Clin Microbiol Infect. 2011; 17: 1135-1141.

24. Nabarro LEB, Shankar C, Pragasam AK, Mathew G, Jeyaseelan V, Veeraraghavan B et al. Clinical and bacterial risk factors for mortality in children with carbapenemresistant Enterobacteriaceae bloodstream infections in India. Pediatr Infect Dis J. 2017; 36: e161-e166.

25. Hsu AJ, Tamma PD. Treatment of multidrug-resistant gramnegative infections in children. Clin Infect Dis. 2014; 58: 1439-1448.

Correspondencia:

David Aguilera-Alonso

Hospital General Universitario Gregorio Marañón

Calle O'Donnell, 48, 28009, Madrid.

Número de teléfono: +34 606663030

E-mail: david.aguilera@salud.madrid.org 